\title{
Magic Numbers in Small Iron Clusters: A First-Principles Study
}

\author{
Eunja Kim ${ }^{\mathrm{a}, *}$, Andrew Mohrland ${ }^{\mathrm{a}}$, Philippe F. Weck ${ }^{\mathrm{b}}$, Tao Pang ${ }^{\mathrm{a}}$, Kenneth R. Czerwinski ${ }^{\mathrm{c}}$, David Tománek ${ }^{\mathrm{d}}$ \\ ${ }^{a}$ Department of Physics and Astronomy, University of Nevada Las Vegas, 4505 Maryland Parkway, Las Vegas, NV 89154, USA \\ ${ }^{b}$ Sandia National Laboratories, P.O. Box 5800, Albuquerque, NM 87185, USA \\ ${ }^{c}$ Department of Chemistry, University of Nevada Las Vegas, 4505 Maryland Parkway, Las Vegas, NV 89154, USA \\ ${ }^{d}$ Physics and Astronomy Department, Michigan State University, East Lansing, Michigan 48824, USA
}

\begin{abstract}
We perform ab initio spin-polarized density functional calculations of $\mathrm{Fe}_{n}$ aggregates with $n \leq 17$ atoms to reveal the origin of the observed magic numbers, which indicate particularly high stability of clusters with 7, 13 and 15 atoms. Our results clarify the controversy regarding the ground state geometry of clusters such as $\mathrm{Fe}_{5}$ and indicate that magnetism plays an important role in determining the stability and magic numbers in small iron clusters.
\end{abstract}

Keywords: Clusters, magic numbers, magnetism

PACS: 82.30.Nr, 68.65.-k, 61.46.Bc

\section{Introduction}

In finite-size atomic aggregates, dubbed quantum dots, equilibrium arrangement of atoms and electronic structure differ fundamentally from the bulk. Similar to atomic nuclei, the stability of alkali atom aggregates is dominated by electronic shell structure, yielding magic numbers $n=2,8,20$, and 40 for the atomic size of particularly abundant clusters [1]. Magnetic interactions add one more layer of complexity and are expected to cause uncommon atomic arrangements and superparamagnetism in clusters of open-shell $3 d$ elements such as Fe. Indeed, significantly enhanced magnetic moments in the range $2.7-3.3 \mu_{\mathrm{B}}$ per atom, much larger than the bulk value of $2.15 \mu_{\mathrm{B}}$, have been observed in small $\mathrm{Fe}_{n}$ clusters [2, 3, 4]. Time-offlight (TOF) mass spectra reveal an unusual sequence of magic numbers $n=7,13,15,19$ and 23 for $\mathrm{Fe}_{n}$ clusters [5], which can be explained neither by electronic shell closure[1] nor by packing of hard spheres [6]. The intimate relationship between structure, magnetic properties and stability poses an uncommon challenge that motivated many theoretical studies of $\mathrm{Fe}_{n}$ clusters $[7,8,9,10,11,12,13,14,15,16,17]$. Still, important controversies remain unresolved among the reported results, including the ground-state atomic arrangements,

\footnotetext{
${ }^{*}$ Corresponding author.

Email address: kimej@physics . unlv.edu (Eunja Kim)
}

magnetic moments, noncollinear magnetism, and finally the stability spectrum of small Fe aggregates.

In an effort to reconcile reported data and obtain microscopic understanding of these systems, we performed $a b$ initio spin-polarized density functional calculations for $\mathrm{Fe}_{n}$ clusters with up to $n=17$ atoms. In particular, we have performed a systematic and extensive search for the ground-state structure of each cluster and computed the corresponding binding energies. All the iron cluster structures previously reported in the literature were considered in this work, in an effort to resolve some of the contradictory findings regarding possible ground-state structures. Computed second derivatives of the binding energy, reflecting relative stabilities, yield magic numbers in agreement with the observed TOF spectra [5]. Our results clarify the controversy regarding the ground state geometry of clusters such as $\mathrm{Fe}_{5}$ and shed new light on the importance of local magnetic moments on the total energy. Good agreement is also obtained between the magnetic moments calculated in this study and available measurements for $\mathrm{Fe}_{n}(n=15-17)$.

\section{Computational methods}

We performed first-principles total energy calculations using the spin-polarized density functional theory (DFT) as implemented in the Vienna $A b$ initio Simulation Package (VASP) [18]. The exchange-correlation 
energy was calculated using the generalized gradient approximation [19] (GGA), with the parameterization of Perdew and Wang [20] (PW91). We performed comparative calculations using the Perdew-Burke-Ernzerhof (PBE) exchange-correlation functional [21] and found only insignificant differences with our PW91-based results. For transition-metal cluster computations, pure exchange-correlation functionals such as PW91 are generally preferred over hybrid functionals, which are less accurate in describing metal-metal bonds [14, 22].

The interaction between valence electrons and ionic cores was described by the Projector Augmented Wave (PAW) method [23, 24]. We solved Kohn-Sham equations using the blocked Davidson iterative matrix diagonalization scheme followed by the residual vector minimization method. The plane-wave cutoff energy for electronic wavefunctions was set at $500 \mathrm{eV}$. A supercell approach was adopted in the calculations, using large cubic unit cells with an edge length of $12 \AA$. Interactions between neighboring clusters were suppressed efficiently in this way, justifying the adequacy of sampling the Brillouin zone by a single $k$-point at $\Gamma$. Fully non-collinear magnetic structure calculations were also carried out with VASP for selected clusters using the default $(0,0,1)$ spin quantization axis in the cubic simulation cell. However, no significant differences with collinear magnetic results were found, in line with recently reported theoretical results [17]. Therefore, only collinear magnetic results are discussed in this study. Geometries were optimized from different starting geometries without symmetry constraints with the conjugate gradient method, accelerated using the Methfessel-Paxton Fermi-level smearing [25] with a width of $0.2 \mathrm{eV}$. The convergence tolerances imposed on the molecular system were $10^{-3} \mathrm{eV}$ for the total energy and $0.01 \mathrm{eV} / \AA$ for the Hellmann-Feynman forces acting on atoms. The accuracy and convergence of our results were checked thoroughly and extensively. We found that applying stricter convergence tolerances did not change our findings.

\section{Results and discussion}

Optimized geometries of the lowest-energy structures of $\mathrm{Fe}_{n}$ clusters with $n=3-17$ are displayed in Fig. 1 along with their symmetry information. The pointgroup symmetry of each cluster was determined using a tolerance of $0.1 \AA$ between the atomic positions in the computed structure and the symmetrized reference structure. With few exceptions, our calculated structures for $n \leq 7$ agree with published theoretical results $[13,14,15]$. Similar to recently predicted equilibrium

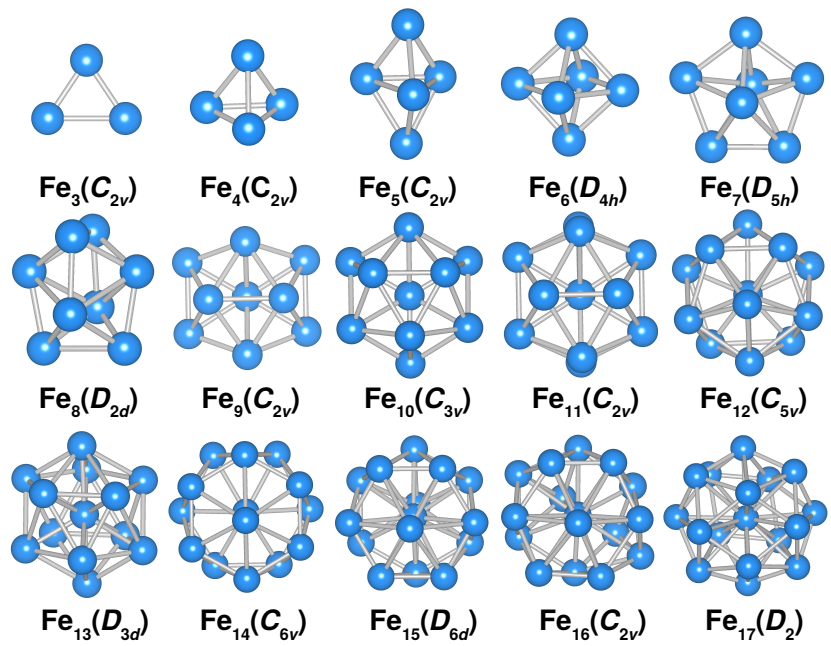

Figure 1: Ball-and-stick models of the equilibrium structures of $\mathrm{Fe}_{n}$ clusters with $n=3-17$ atoms. Molecular point-group symmetries are given in parentheses.

structures $[12,14]$, many of our ground-state geometries experience stabilizing Jahn-Teller distortions that reduce their symmetry.

Relative stability of particular structural isomers is determined by a competition between the tendency to stabilize covalent bonds to the highest degree on one hand, causing close packing, and on the other hand to limit inter-atomic repulsion and maximize the spin polarization energy, which favors open structures. Low average coordination numbers in small atomic aggregates are the main cause of reduced atomic binding energies and interatomic distances. As the average coordination number increases with increasing cluster size, so does the average binding energy and nearest-neighbor distance while gradually approaching bulk values. For the sake of convenience, we define the total binding energy of an $\mathrm{Fe}_{n}$ cluster as $E(n)=n E_{\text {tot }}(\mathrm{Fe}$ atom $)-E_{\text {tot }}\left(\mathrm{Fe}_{n}\right)$ and the average binding energy per atom $E_{\mathrm{b}}(n)=$ $E(n) / n$. Figure 2(a) displays computational results of the current and related studies, documenting the general increase of the atomic binding energy with increasing cluster size $n$, in agreement with published results $[9,13,14,15,16]$. We find that the relationship between $E_{\mathrm{b}}(n)$ and the inverse cluster size $1 / n$ is nearly linear for $n>2$, allowing for an extrapolation to an infinitely large cluster. The extrapolated value $E_{\mathrm{b}}(\infty)=4.35 \mathrm{eV}$ for $1 / n \rightarrow 0$ is close to the experimental value $E_{\mathrm{coh}}=$ $4.28 \mathrm{eV}$ for the body-centered cubic $(b c c)$ phase of bulk $\mathrm{Fe}$ [26], suggesting a uniform trend towards higher stability as the cluster sizes gradually approach the bulk 


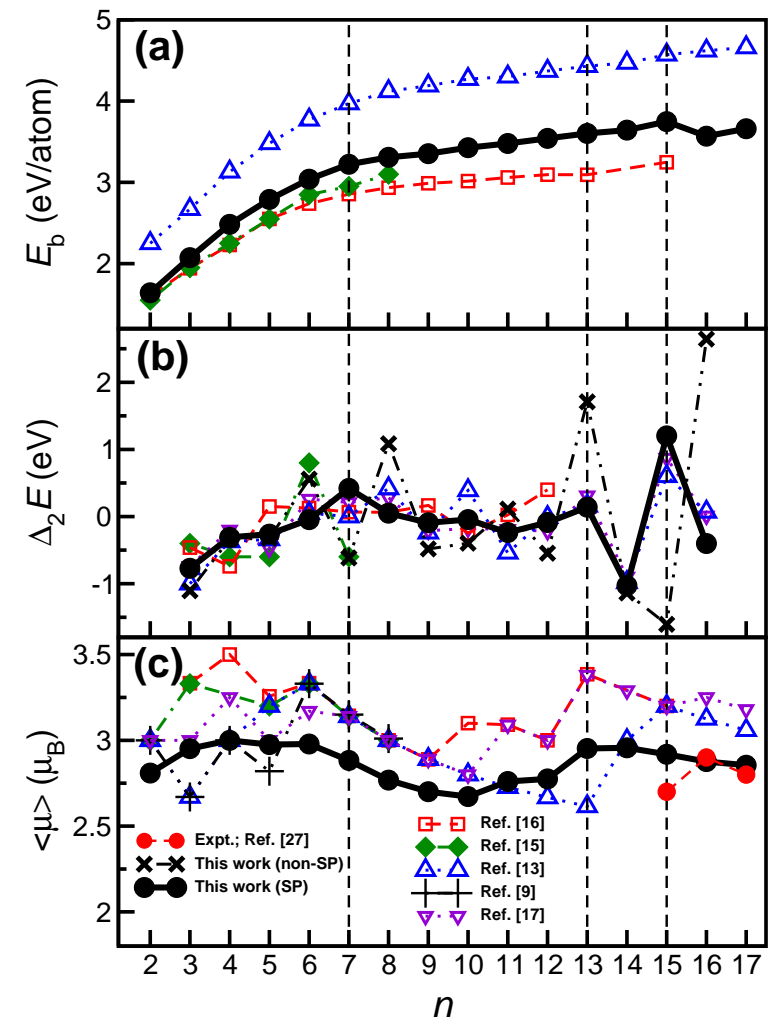

Figure 2: Dependence of (a) the binding energy per atom $E_{b}(n)$, (b) the second energy differences $\Delta_{2} E(n)$, and (c) the average magnetic moment per atom $\langle\mu\rangle(n)$ on the cluster size $n$ of $\mathrm{Fe}_{n}$ clusters in their equilibrium geometry, depicted in Fig. 1 . The vertical dashed lines at $n=7,13$ and 15 indicate observed magic numbers in the mass spectra of Sakurai et al. [5]. Spin-polarized (SP) and non-SP calculations are represented.

limit.

In the following, we will focus our attention on deviations from this uniform trend, which should reveal particularly stable structures that will be associated with magic cluster sizes. We may expect uncommon equilibrium geometries with high relative stability to emerge as most stable due to the dominant role of the directional $3 d$ orbitals in $\mathrm{Fe}_{n}$ clusters.

Abundances in TOF spectra, called magic atomic numbers, are associated with particularly stable structures that are less likely than average to lose or acquire additional atoms in collisions. The corresponding quantity to look for is the second total binding energy difference $\Delta_{2} E(n)=E(n+1)+E(n-1)-2 E(n)$ (see Refs. $[13,15,16,17])$. This difference is a measure of the relative binding energy of a $n$-atom cluster with respect to clusters with $n+1$ and $n-1$ atoms. Therefore, peaks in this function indicate relatively most stable structures that can be associated with magic numbers of atoms. We display our results for $\Delta_{2} E(n)$ as a function of cluster size in Fig. 2(b) along with corresponding results obtained by other groups. These published results disagree strongly with each other, suggesting magic numbers to occur at $n=5,9$ [16], $n=6$ [15], $n=6,8,10,13,15$ [13]. When compared to the experimentally observed magic numbers [5] $n=7,13,15$, many of these studies correctly identify $n=13,15$ as unusually stable, but all of them miss $n=7$ and also list other sizes that are not observed experimentally [17]. Our $\Delta_{2} E(n)$ results in Fig. 2(b) show only three clear peaks, associated with magic numbers at $n=7,13,15$, in complete agreement with the observed TOF spectra [5]. This agreement suggests that structures we identify as equilibrium arrangements may indeed be those found in the experiment. Close inspection of our results in Fig. 2(b) reveals that spin-unpolarized calculations correctly reproduce only the experimental magic number at $n=13$, but differ from the experiment elsewhere, predicting incorrect magic numbers at $n=6,8$, and 11 . This observation suggests that magnetism plays an important role in determining the relative stability of structural isomers of iron clusters.

In the following, we focus our attention on the role of magnetism in determining magic numbers in $\mathrm{Fe}_{n}$ clusters. For clusters of interest, we calculated the effective magnetic moment, which can be compared directly to experimental data. Magnetism may play a stronger role in small clusters than in bulk systems due to the reduced average coordination number. The Stoner model for band magnetism, which has been developed for bulk structures, is not strictly applicable to finite-size clusters. Still, it correctly predicts an increase in the average magnetic moment in small clusters with reduced average coordination number, in agreement with experimental data [2, 3, 4]. Results of this and other recent theoretical studies, depicted in Fig. 2(c), are in general agreement as far as the average magnetic moment $\langle\mu\rangle$ should decrease with increasing cluster size, until reaching the bulk value of $2.15 \mu_{\mathrm{B}}$ per atom. Our results show very good agreement with available experimental data [27] for $n=15-17$, while all the previous computational predictions systematically overestimate these experimental values.

Next we will discuss our results for individual cluster sizes, focussing on the role of magnetism in determining the equilibrium structure and stability. Among the smallest cluster sizes, the equilibrium bond length is shortest in the $\mathrm{Fe}_{2}$ dimer. The calculated value of $d=1.98 \AA$ is close to the experimental estimates of 
$1.87 \pm 0.13$ [28] and $2.02 \pm 0.13 \AA$ [29] reported for $\mathrm{Fe}_{2}$ trapped in argon and neon matrices using extended $\mathrm{X}$-ray absorption fine structure spectroscopy, and are significantly smaller than the bulk nearest-neighbor distance $d=2.49 \AA$. Our computed binding energy, $E_{\mathrm{b}}(2)=1.64 \mathrm{eV}$, slightly overestimates the experimental values [30] ranging between 0.83 and $1.32 \mathrm{eV}$. Spin polarization resulting in the net magnetic moment of $2.81 \mu_{\mathrm{B}}$ per atom contributes significantly to the stability of $\mathrm{Fe}_{2}$, as seen in the total energy difference of $\Delta E(2)=1.10 \mathrm{eV} /$ atom compared to spin-unpolarized calculations. The calculated magnetic moment is in fair agreement with the experimental estimate of $3.3 \pm 0.5 \mu_{\mathrm{B}}$ per atom [10]

We find that the most stable $\mathrm{Fe}_{3}$ isomer is an isosceles triangle $\left(C_{2 v}\right)$ with bond lengths 2.06 and $2.30 \AA$, in good agreement with previous DFT results $[7,8$, $9,13,16,10]$. This structure is $0.0026 \mathrm{eV} /$ atom and $0.003 \mathrm{eV} /$ atom lower in energy than the scalene triangle $\left(C_{s}\right)$ with $2.08 \AA, 2.22 \AA$ and $2.36 \AA$ sides and the equilateral triangle $\left(D_{3 h}\right)$ with $2.22 \AA$ sides, respectively. The calculated average magnetic moment $2.95 \mu_{\mathrm{B}}$ is consistent with the experimental value of $2.7 \pm 0.3 \mu_{\mathrm{B}}[4]$ and stabilizes the structure by $\Delta E(3)=$ $1.04 \mathrm{eV} /$ atom. The most stable $\mathrm{Fe}_{4}$ cluster is a tetrahedron, consisting of four isocsceles triangles with bond lengths of $2.24 \AA$ and $2.48 \AA$, which is $0.007 \mathrm{eV} /$ atom lower in energy than the one of equilateral triangles with bond lengths of $2.28 \AA$. Our finding is consistent with previous results $[7,8,9,10,13,16]$.

The most stable structure of $\mathrm{Fe}_{5}$ is a trigonal bipyramid (TBP) with $C_{2 v}$ symmetry and collinear spinup magnetic moments for all atoms. This follows a long-standing controversy concerning the geometric and magnetic structure of this cluster. Our calculations with noncollinear ferromagnetic arrangement of the local spins resulted in the same symmetry with a negligible energy stabilization of $0.006 \mathrm{eV} /$ atom. After initial indications of a ferromagnetic spin arrangement, planewave pseudopotential LSDA calculations suggested that the ground state of $\mathrm{Fe}_{5}$ was a distorted triangular bipyramid with noncollinear arrangement of local spins [10]. These findings were corroborated by Hobbs et al. in Ref. [11], who predicted a noncollinear ground state for $\mathrm{Fe}_{5}$ with a total magnetic moment of $14.5 \mu_{\mathrm{B}}$ in their DFT-LSDA study of noncollinear magnetism. Subsequent DFT studies based on the generalized gradient approximation (GGA) have since suggested that the $D_{3 h}$ isomer of $\mathrm{Fe}_{5}$ with noncollinear magnetic arrangment and a total magnetic moment of $15.9 \mu_{\mathrm{B}}$ is a metastable structure that spontaneously converts to a distorted TBP [12] with ferromagnetic spin arrangement and a total magnetic moment of $16.0 \mu_{\mathrm{B}}$. Other competing geometries, including pyramidal structures with square and diagonal bases, are less stable.

The most stable isomer of $\mathrm{Fe}_{6}$ is an octahedron with $D_{4 h}$ symmetry, in good agreement with previous DFT studies $[10,12,13,15,16]$, but contrasting with the findings of Ballone and Jones [9], who found that a capped TBP $\left(C_{2 v}\right)$ structure is most stable. We found the later to be $0.003 \mathrm{eV} /$ atom higher than the former. We also found the pentagonal bipyramid (PBP) in $D_{5 h}$ symmetry to be the most stable geometry for $\mathrm{Fe}_{7}$, with all spins in a ferromagnetic arrangement, in good agreement with previous DFT studies [7, 8, 9, 10, 12, 13, 16]. The high relative stability of this structure with respect to its neighbors of different size causes its dominance as a magic cluster size in mass spectrometry data [5]. The calculated bond lengths are $2.30 \AA$ and $2.40 \AA$, in good agreement with previous DFT studies $[9,13]$. The pronounced difference between our spin-polarized and spin-unpolarized results suggests that magnetism contributes significantly to $n=7$, stablizing the structure by $0.82 \mathrm{eV} /$ atom and becoming a magic number.

The computed stable structure of $\mathrm{Fe}_{8}$ is a bisdisphenoid structure with $D_{2 d}$ symmetry, consistent with previous DFT studies $[12,13,15,16,17]$. The calculated magnetic moments of this $D_{2 d}$ structure are all positive, with $\langle\mu\rangle=2.77 \mu_{\mathrm{B}}$, and the bond lengths are in the range $2.29-2.56 \AA$. The bi-capped octahedron $\left(D_{2 d}\right)$ is 0.08 $\mathrm{eV} /$ atom higher than the bisdisphenoid structure. $\mathrm{Fe}_{9}$ isomers include a four-capped TBP $\left(C_{2 v}\right)$, a tri-capped trigonal prism $\left(D_{3 h}\right)$ [13], a single capped square antiprism $\left(C_{4 v}\right)[12,17]$, and a $b c c\left(O_{h}\right)$ structure. We found that the most stable isomer among them is the four-capped TBP (or a bi-capped PBP) in $C_{2 v}$ symmetry, consistent with the findings of Ma et al. [16] but differs from previous DFT results [12, 13, 17]. Our calculations predict that the $\mathrm{Fe}_{9}$ isomers in $\mathrm{D}_{3 h}, C_{4 v}$, and $O_{h}$ are $0.08,0.06$, and $0.08 \mathrm{eV} /$ atom, respectively, less stable than the ground state $C_{2 v}$ structure. The magnetic moments for all atoms are spin-up, with $\langle\mu\rangle=2.70 \mu_{\mathrm{B}}$, and the bond lengths are in the range $2.32-2.50 \AA$.

Our finding that the most stable $\mathrm{Fe}_{10}$ structure is a three-capped PBP stucture $\left(C_{3 v}\right)$ differs from previous DFT results $[12,13,17]$ suggesting a bi-capped square antiprism geometry $\left(D_{4 d}\right)$, but agrees with the findings of Ma et al. [16]. Our calculations show that the $\mathrm{Fe}_{10}$ isomer with $D_{4 d}$ symmetry is $0.03 \mathrm{eV} /$ atom less stable than the ground state $C_{3 v}$ geometry. The calculated average magnetic moment is $\langle\mu\rangle=2.67 \mu_{\mathrm{B}}$, and the bond lengths are in the range $2.26-2.42 \AA$. The lowest-energy $\mathrm{Fe}_{11}$ structure is a tetra-capped PBP with $C_{2 v}$ symmetry, in agreement with previously reported DFT results 
$[13,16,17]$. The bond lengths range from $2.24 \AA$ to $2.60 \AA$ and the calculated average magnetic moment is $\langle\mu\rangle=2.76 \mu_{\mathrm{B}}$.

The most stable $\mathrm{Fe}_{12}$ structure adopts the $C_{5 v}$ symmetry following an icosahedral growth pattern as shown in Fig. 1, in accord with most of previous DFT studies $[13,16,17]$. The $\mathrm{Fe}_{12}$ isomer with $I_{h}$ symmetry has bond lengths of $2.38-2.40 \AA$ and is $0.03 \mathrm{eV} /$ atom less stable than the $C_{5 v}$ structure with bond lengths of $2.27-2.74 \AA$. The calculated average magnetic moment is $\langle\mu\rangle=2.78 \mu_{\mathrm{B}}$. The distorted icosahedral structure $\left(D_{3 d}\right)$ is the most stable atomic arrangement in $\mathrm{Fe}_{13}$, making this size a magic number, in agreement with most of previously reported DFT results $[12,13,16,17]$. The $I_{h}$ structure with bond lengths of $2.34-2.65 \AA$ is $0.005 \mathrm{eV} /$ atom less stable than the $D_{3 d}$ equilibrium geometry. The calculated average magnetic moment is $\langle\mu\rangle=2.95 \mu_{\mathrm{B}}$. According to Fig. 2(c), this value is higher than in similar-sized clusters, stablizing the structure by $0.68 \mathrm{eV} /$ atom and contributing to making $n=13$ a magic number.

The most stable $\mathrm{Fe}_{14}$ and $\mathrm{Fe}_{15}$ clusters both possess a 6-fold symmetry axis based on the centered hexagonal antiprism (HAP). It is a single capped HAP for the $\mathrm{Fe}_{14}$ isomer, in agreement with Dieguez et al. [13]. The $\mathrm{Fe}_{14}$ isomer based on the $\mathrm{Fe}_{13}$ isomer with $I_{h}$ symmetry [17], just replacing a five-member ring by six-member ring, is $0.002 \mathrm{eV} /$ atom less stable than the ground state geometry in this study. The bond lengths in the $C_{6 v}$ isomer of $\mathrm{Fe}_{14}$ range from $2.33-2.72 \AA$, and the average magnetic moment is $\langle\mu\rangle=2.96 \mu_{\mathrm{B}}$. The optimum structure of the $\mathrm{Fe}_{15}$ cluster is a bi-capped centered HAP with $D_{6 d}$ symmetry, consistent with most of previous studies [12, 13, 16, 17], with bond lengths ranging from $2.29-2.60 \AA$ and the average magnetic moment $\langle\mu\rangle=2.92 \mu_{\mathrm{B}}$. The large difference between our spin-unpolarized and spin-polarized results for $\mathrm{Fe}_{15}$ in Fig. 2(b) is a strong indication that magnetism contributes significantly to this cluster size, stablizing the structure by $0.82 \mathrm{eV} /$ atom and becoming a magic number.

For $\mathrm{Fe}_{16}$ and $\mathrm{Fe}_{17}$ we found the most stable isomers to be based on the $\mathrm{Fe}_{15}$ structure consisting of two sixmember rings with singly and doubly capped atoms for $\mathrm{Fe}_{16}$ and $\mathrm{Fe}_{17}$, respectively, in agreement with the previously reported DFT results $[13,17]$. The lowest-energy $\mathrm{Fe}_{16}$ isomer adopts a $C_{2 v}$ symmetry with bond distances varying between $2.25-2.90 \AA$ and $\langle\mu\rangle=2.88 \mu_{\mathrm{B}}$. According to our calculations, the most stable $\mathrm{Fe}_{17}$ structure with $D_{2}$ symmetry, is characterized by bond lengths in the range $2.28-2.94 \AA$, and has an average magnetic moment of $2.85 \mu_{\mathrm{B}}$. Even though Dieguez et al. and
Gutsev et al. proposed different ways to bi-capped the $\mathrm{Fe}_{15}$ structure to form the $\mathrm{Fe}_{17}$ isomer their energy difference is less than $0.001 \mathrm{eV} /$ atom, thus negligible. According to Fig. 2(a), both $\mathrm{Fe}_{16}$ and $\mathrm{Fe}_{17}$ are less stable than $\mathrm{Fe}_{15}$.

The above findings for most stable isomers are in agreement with intuition: three-dimensional structures with closer packing are generally more stable. The overall trend for the average magnetic moment $\langle\mu\rangle$ as a function of Fe cluster size mirrors the experimental findings [2, 27], showing that average magnetic moments for small iron clusters are in the range $2.7-3.3 \mu_{\mathrm{B}}$, oscillating around $\approx 3 \mu_{\mathrm{B}}$, and slowly converging towards the bulk value of $\langle\mu\rangle=2.15 \mu_{\mathrm{B}}$ in clusters with more than 25 atoms $[3,4]$.

\section{Summary and Conclusions}

In summary, we have performed $a b$ initio spinpolarized density functional calculations of $\mathrm{Fe}_{n}$ aggregates with $n \leq 17$ atoms to reveal the origin of the observed magic numbers. Our results suggest particularly high stability of clusters with 7,13 and 15 atoms, in agreement with observed mass spectra, but at variance with previous studies. Our results have helped us to clarify the controversy regarding the ground state geometry of clusters such as $\mathrm{Fe}_{5}$ and indicate that magnetism plays an important role in determining the stability and magic numbers in small iron clusters.

\section{Acknowledgments}

EK acknowledges funding through subcontract with Los Alamos National Laboratory within the Department of Energy's Fuel Cycle R\&D program. Sandia National Laboratories is a multi-program laboratory managed and operated by Sandia Corporation, a wholly owned subsidiary of Lockheed Martin Corporation, for the U.S. Department of Energy's National Nuclear Security Administration under contract DE-AC0494AL85000. DT was supported by the National Science Foundation Cooperative Agreement \#EEC-0832785, titled "NSEC: Center for High-rate Nanomanufacturing". A portion of the research was performed using high performance computing resources at EMSL, a DOE Office of Science User Facility sponsored by the Office of Biological and Environmental Research and located at Pacific Northwest National Laboratory. We thank Drs. G.L. Gutsev, D. Jeffery, V. Kwong, S. Lepp, M. Pravica, and B. Zygelman for useful discussions. 


\section{References}

[1] W. D. Knight, K. Clemenger, W. A. de Heer, W. A. Saunders, M. Y. Chou, M. L. Cohen, Phys. Rev. Lett. 52 (1984) 2141.

[2] I. M. L. Billas, J. A. Becker, A. Châtelain, W. A. de Heer, Phys. Rev. Lett. 71 (1993) 4067

[3] I. M. L. Billas, A. Châtelain, W. A. de Heer, Science 265 (1994) 1682.

[4] D. M. Cox, D. J. Trevor, R. L. Whetten, E. A. Rohlfing, A. Kaldor, Phys. Rev. B 32 (1985) 7290.

[5] M. Sakurai, K. Watanabe, K. Sumiyama, K. Suzuki, J. Chem. Phys. 111 (1999) 235.

[6] W. Miehle, O. Kandler, T. Leisner, O. Echt, J. Chem. Phys. 91 (1989) 5940.

[7] J. L. Chen, C. S. Wang, K. A. Jackson, M. R. Pederson, Phys. Rev. B 44 (1991) 6558.

[8] M. Castro, D. R. Salahub, Phys. Rev. B 49 (1994) 11842.

[9] P. Ballone, R. O. Jones, Chem. Phys. Lett. 233 (1995) 632.

[10] T. Oda, A. Pasquarello, R. Car, Phys. Rev. Lett. 80 (1998) 3622.

[11] D. Hobbs, G. Kresse, J. Hafner, Phys. Rev. B 62 (2000) 11556.

[12] G. Rollmann, P. Entel, S. Sahoo, Comput. Mater. Sci. 35 (2006) 275.

[13] O. Diéguez, M. M. G. Alemany, C. Rey, P. Ordejón, L. J. Gallego, Phys. Rev. B 63 (2001) 205407.

[14] G. L. Gutsev, J. C. W. Bauschlicher, J. Phys. Chem. A 107 (2003) 7013.

[15] S. Yu, S. Chen, W. Zhang, L. Yu, Y. Yin, Chem. Phys. Lett. 446 (2007) 217.

[16] Q. M. Ma, Z. Xie, J. Wang, Y. Liu, Y. C. Li, Solid State Commun. 142 (2007) 114.

[17] G. L. Gutsev, C. A. Weatherford, P. Jena, E. Johnson, B. R. Ramachandran, J. Phys. Chem. A 116 (2012) 10218.

[18] G. Kresse, J. Furthmüller, Phys. Rev. B 54 (1996) 11169.

[19] J. P. Perdew, J. A. Chevary, S. H. Vosko, K. A. Jackson, M. R. Pederson, D. J. Singh, C. Fiolhais, Phys. Rev. B 46 (1992) 6671.

[20] J. P. Perdew, Y. Wang, Phys. Rev. B 45 (1992) 13244.

[21] J. P. Perdew, K. Burke, M. Ernzerhof, Phys. Rev. Lett. 77 (1996) 3865 .

[22] F. Furche, J. P. Perdew, J. Chem. Phys. 124 (2006) 044103.

[23] P. E. Blöchl, Phys. Rev. B 50 (1994) 17953.

[24] G. Kresse, D. Joubert, Phys. Rev. B 59 (1999) 1758

[25] M. Methfessel, A. T. Paxton, Phys. Rev. B 40 (1989) 3616.

[26] C. Kittel, Introduction to Solid State Physics. Wiley and Sons: New York, 1986

[27] M. B. Knickelbein, Chem. Phys. Lett. 353 (2002) 221.

[28] P. A. Montano, G. K. Shenoy, Solid State Commun. 35 (1980) 53.

[29] H. Purdum, P. A. Montano, G. K. Shenoy, T. Morrison, Phys. Rev. B 25 (1982) 4412.

[30] P. J. Brucat, L.-S. Zheng, C. L. Pettiette, S. Yang, R. E. Smalley, J. Chem. Phys. 84 (1986) 3078. 


\section{Graphical abstract}

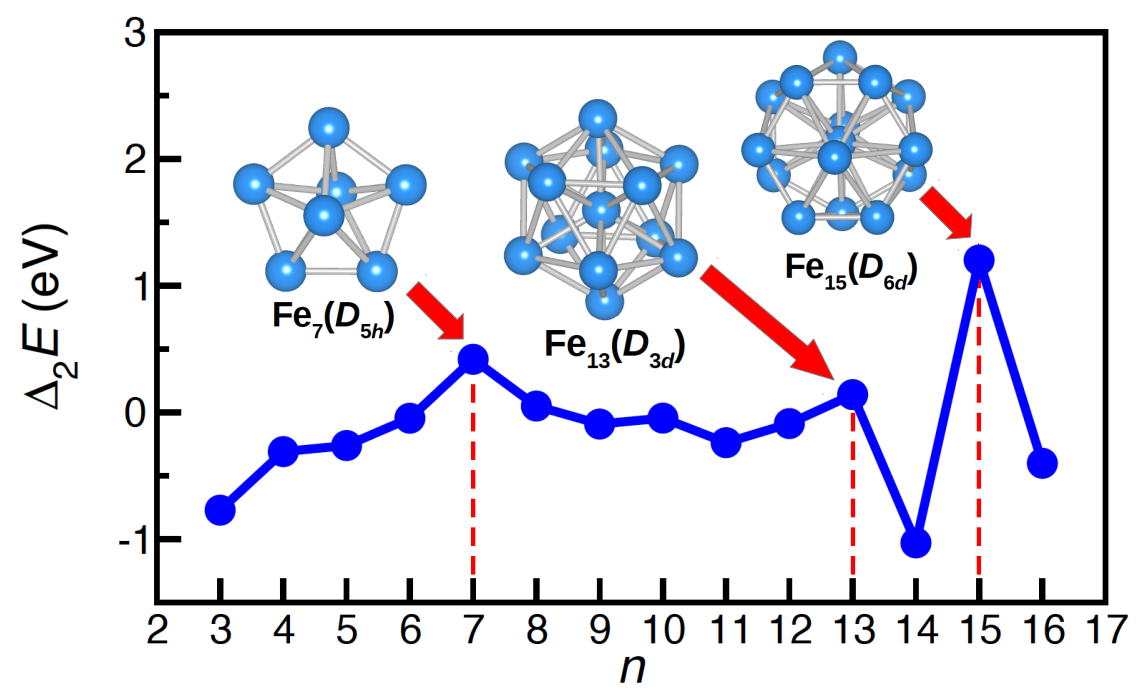

